\title{
Cancer stem-like side population cells in the human nasopharyngeal carcinoma cell line CNE-2 possess epithelial mesenchymal transition properties in association with metastasis
}

\author{
DAN GUO $^{1}$, BEN-LING XU ${ }^{2}$, XU-HUA ZHANG ${ }^{2}$ and MING-MIN DONG ${ }^{1}$ \\ ${ }^{1}$ Department of Otolaryngology, Head and Neck Surgery, The First Affiliated Hospital, Zhengzhou University, \\ Zhengzhou, Henan 450052; ${ }^{2}$ Department of Biotherapy, Laboratory Center, Affiliated Cancer \\ Hospital, Zhengzhou University, Zhengzhou, Henan 450008, P.R. China
}

Received February 2, 2012; Accepted March 6, 2012

DOI: $10.3892 /$ or.2012.1781

\begin{abstract}
It has been recently reported that side population (SP) cells in nasopharyngeal carcinoma (NPC) cell lines display characteristics of cancer stem-like cells. However, the biological behavior and the significance of these cells for NPC progression remain unclear. In this study, we isolated SP cells from the NPC cell line CNE-2 by flow cytometry and investigated their biological characteristics. We discovered that SP cells had stronger colony forming abilities compared to the non-side population (NSP) cells, and observed that some SP cells looked more like the shape of mesenchymal cells when cultured in the common polyHEMA-coated flask. When checked by quantitative real-time PCR, the SP cells expressed higher levels of stemness-related genes Oct4, Sox 2 and Nanog, and mesenchymal cell-related genes $\mathrm{N}$-cadherin, vimentin and Snail, while they expressed lower levels of the epithelial cell-related gene, E-cadherin. Western blot and immunofluorescence staining methods further verified that SP cells expressed higher vimentin and expressed lower E-cadherin levels. Finally, Transwell invasion assay results indicated that the SP cells had higher invasive potential compared to NSP cells. Collectively, our data reveal that SP cells in the CNE-2 cell line not only possess the properties of cancer stem cells, but also have more mesenchymal cell characteristics which are associated with epithelial mesenchymal transition (EMT) and cancer cell invasion and metastasis. These findings are helpful for developing novel targets for effective clinical treatment of NPC.
\end{abstract}

\section{Introduction}

Nasopharyngeal carcinoma (NPC) is fairly rare among Caucasians in Western Europe and North America $(1 / 100,000)$,

Correspondence to: Professor Ming-Min Dong Department of Otolaryngology, Head and Neck Surgery, the First Affiliated Hospital, Zhengzhou University, Zhengzhou, Henan 450052, P.R. China

E-mail:dmment@163.com

Key words: nasopharyngeal carcinoma, cancer stem cells, epithelialmesenchymal transition, metastasis but it is the most frequent head and neck tumor in Guangdong, south China, where the incidence peaks at 50/100,000 $(1,2)$. NPC is a highly malignant tumor which often invades adjacent regions and metastasizes to regional lymph nodes and distant organs. Thirty to sixty percent of patients with NPC will eventually develop distant metastases. Although advances in therapy have improved the quality of life, survival rates have remained unchanged over the past decades. Mortality from this disease remains high because of the development of distant metastases and the emergence of local and systemic recurrences resistant to chemoradiotherapy. It is therefore essential to develop a deeper understanding of the cellular and molecular mechanisms of NPC metastasis and their recurrence in order to develop more effective therapeutic approaches.

Evidence has recently been accumulating to support the hypothesis that tumors contain a small subpopulation of cells called cancer stem cells (CSC), which exhibit self-renewing capacities and are responsible for tumor maintenance and metastasis (3). Currently, there are mainly two methods used to identify and isolate cancer stem cells. One is to use the molecular markers expressed on the cell surface by flow cytometry or immune magnetic beads. Several molecules have been identified as potential cancer stem cells markers, such as CD133 in brain tumors, colorectal carcinoma, prostatic carcinoma and pancreatic carcinoma (4-7) and CD44 in head and neck, breast, and ovarian cancer (8-10). However, these findings are still controversial. For example, CD133 has been challenged as a cancer stem cell marker in colon cancer (11), while CD44 has been questioned as a head and neck cancer stem cell marker (12). In head and neck squamous cell carcinoma (HNSCC), Prince et al first demonstrated that a CD44 ${ }^{+}$ population of cells possessed the properties of CSC (8), but a relatively high number of these cells (5,000 cells) were needed to generate new tumors in immunodeficient mice, indicating either a low frequency of CSC or a low specificity of CD44 as a CSC-marker in HNSCC. The latter hypothesis is supported by the observation that CD44s and CD44v6 expression levels do not distinguish normal from benign or malignant epithelia of the head and neck. CD44s and CD44v6 are abundantly expressed in the majority of cells in head and neck tissues, including carcinomas (12). Thus, the identification of more 
specific CSC markers for HNSCC is desirable. The second method using the nucleic acid dye Hoechst 33342 has identified so-called side population cells (SP) with stem cell characters. Stem cells can pump out the dye from the cytoplasm due to the activity of ABC transporter transmembrane proteins. SP cells are a distinct, small cell population composed of unstained cells in the left lower quadrant of a flow activated cell sorter (FACS) profile (13). This method is mainly used for cultured tumor cells without known stem cell markers.

The epithelial-mesenchymal transition (EMT) is characterized as a switch from a polarized epithelial phenotype to a highly motile fibroblast or mesenchymal phenotype. During EMT, epithelial cells break down cell to cell and cell extracellular matrix contacts and migrate to other locations in the body (14). EMT is critical to metazoan embryogenesis, chronic inflammation and fibrosis, and has been demonstrated to be a central mechanism in cancer invasiveness and metastasis (15). It has been reported that EMT generates cells with stem celllike properties (16), and that EMT often occurs in cancer stem cells $(17,18)$, which suggests that metastases are sometimes caused by cancer cells that acquire stem cell characteristics.

Wang et al have reported that SP cells in the CNE-2 human NPC cell line display stem cell characteristics (19). However, the molecular mechanisms underlying the regulation of SP cells in NPC remain unclear. In the present study, we examined whether SP cells of the CNE-2 NPC cell line have both characteristics of cancer stem cells and EMT. We report evidence that SP cells enrich CSC-like cells and have the properties of mesenchymal cells.

\section{Materials and methods}

Analyzing and sorting of SP cells by flow cytometry. The human NPC cell line CNE-2 was cultured in the RPMI-1640 medium (Gibco, USA) supplemented with $10 \%$ fetal bovine serum (FBS) (Gibco), $100 \mathrm{U} / \mathrm{ml}$ penicillin $\mathrm{G}$, and $100 \mu \mathrm{g} / \mathrm{ml}$ streptomycin, maintained at $37^{\circ} \mathrm{C}$ in a humidified $5 \% \mathrm{CO}_{2}$ incubator. When the cells grew about $80 \%$ confluent in the flask, they were analyzed by flow cytometry (FACSAria II, BD Biosciences). Cells were digested with $0.25 \%$ trypsin and $1 \mathrm{mM}$ EDTA, washed with calcium/magnesium free phosphate-buffered saline (PBS), resuspended in RPMI-1640 (supplemented with $2 \%$ FBS) with a concentration of $1 \times 10^{6}$ cells $/ \mathrm{ml}$. Subsequently, the DNA-binding dye Hoechst 33342 (Sigma, St. Louis, MO) was added at a final concentration of $5 \mu \mathrm{g} / \mathrm{ml}$, with or without verapamil (Sigma) as a confirmation at a final concentration of $100 \mu \mathrm{mol} / 1$. Both cell treatment groups were incubated at $37^{\circ} \mathrm{C}$ in a $5 \% \mathrm{CO}_{2}$ incubator for $90 \mathrm{~min}$ in the dark with every $30 \mathrm{~min}$ interval mixing. After incubation, cells were washed with ice-cold PBS and filtered through a $45 \mu \mathrm{m}$ cell strainer to obtain single-suspension cells. Five minutes before the analysis of cells and sorting using FACS AriaII, a final concentration of $1 \mu \mathrm{g} / \mathrm{ml}$ propidium iodide (PI) (Sigma) was added. Hoechst 33342 was excited with a UV laser at $350 \mathrm{~nm}$ and fluorescence emission was measured with 405/ BP309 (Hoechst blue) and 570/BP20 (Hoechst red) optical filters. PI labeling was measured through the 630/BP30 filter for the discrimination of dead cells. PI-negative cells were sorted into two subpopulations, Hoechst 33342 negative cells, SP cells, whose ability of fluorescent efflux could be blocked by verapamil, and Hoechst 33342 positive cells (non-SP cells). Finally, the two groups were collected for sorting purity evaluation and further experiments.

Clone formation assay. After FACS sorting, the SP and NSP cells were incubated in 6-well plates at a density of 500 cells per well in RPMI-1640 medium supplemented with $10 \%$ FBS. Cells were cultivated under standard culture conditions. After most cell clones had expanded to more than 50 cells (about 12 days), they were washed twice with PBS, fixed in methanol for $15 \mathrm{~min}$, and stained with crystal violet for $15 \mathrm{~min}$ at room temperature. After washing out the dye, the number of clones which contained more than 50 cells was counted microscopically and the results were compared. The clone formation efficiency (CFE) was the ratio of the formation of clone numbers to the planted cell numbers.

Quantitative real-time RT-PCR. Total-RNA was extracted by using the RNAsimple total-RNA kit (Invitrogen Life Technologies, Carlsbad, CA), then converted to cDNA with the Quantscript RT kit (Invitrogen). qRT-PCRs were carried out in a $20 \mu \mathrm{l}$ final volume using Power SYBR-Green Mix (Takara, Bio, Inc., Shiga, Japan) and run on a 7300 Real-Time PCR System (Applied Biosystems, USA). Reactions were carried out in triplicate as controls and the housekeeping gene GAPDH was used as an internal control to normalize the expression levels of different genes. Their reaction condition was: initiation with a $10 \mathrm{sec}$ denaturation at $95^{\circ} \mathrm{C}$, followed by 40 cycles of amplification with $10 \mathrm{sec}$ of denaturation at $95^{\circ} \mathrm{C}$, $5 \mathrm{sec}$ of annealing according to the melting temperatures of each pair of primers and $30 \mathrm{sec}$ of extension at $60^{\circ} \mathrm{C}$. Then data were analyzed using the modified $\Delta \Delta \mathrm{Ct}$ method (20). The genes examined and their PCR primers are listed in Table I.

Western blotting. Fresh-sorted cells of SP and NSP were collected respectively. Cells were lysed in the Nonidet P-40 lysis buffer containing $50 \mathrm{mM}$ Tris- $\mathrm{HCl}(\mathrm{pH} 7.5), 150 \mathrm{mM} \mathrm{NaCl}, 1 \%$ Nonidet P-40, $1 \mathrm{mM}$ PMSF, $1 \mathrm{mM}$ DTT, $10 \mathrm{U} / \mathrm{ml}$ aprotinin, and $20 \mu \mathrm{g} / \mathrm{ml}$ leupeptin. Cell lysates were cleared by centrifugation, and protein concentrations were determined using the BCA protein assay (Pierce, USA). Equal amounts of protein sample $(20 \mu \mathrm{g})$ were separated on $4 \%$ SDS acrylamide gel (Bio-Rad, USA) for $0.5 \mathrm{~h}$ at $60 \mathrm{~V}$ and on $12 \%$ SDS acrylamide gel for $1.5 \mathrm{~h}$ at $150 \mathrm{~V}$. The samples were then transferred onto a nitrocellulose membrane for $1.5 \mathrm{~h}$ at $20 \mathrm{~V}$ (Whatman, UK). After blocking in 5\% fat-free milk, the membrane was incubated with the primary antibody (anti-E-cadherin mouse monoclonal antibody, 1:3,000; anti-vimentin mouse monoclonal antibody, (BD Biosciences, USA) 1:4,000 overnight at $4^{\circ} \mathrm{C}$, followed by incubation with the secondary HRP-conjugated goat antimouse $\operatorname{IgG}$ antibody for $1 \mathrm{~h}$ at room temperature $(1: 5,000)$. The antibody was diluted in PBS containing 5\% Blotto (Santa Cruz Biotechnology, Inc. Santa Cruz, CA) and 0.1\% Tween-20. The stained membranes were visualized by enhanced chemiluminescence reaction using the ECL Plus kit (GE Healthcare, USA). The mouse monoclonal $\beta$-actin antibody (BD Biosciences) was used as the loading control for western blot analysis at a dilution of 1:5,000. The band intensities of the PCR products were analyzed using the UVP VisionWorks LS $6.6 \alpha$ (UVP), and were expressed as the means \pm SD. 
Table I. Examined genes and their PCR primers.

\begin{tabular}{|c|c|c|}
\hline Gene & Primer sequence $\left(5^{\prime} \rightarrow 3^{\prime}\right)$ & Size of product (bp) \\
\hline ABCG2 & $\begin{array}{l}\text { F: GGATGAGCCTACAACTGGCTT } \\
\text { R: CTTCCTGAGGCCAATAAGGTG }\end{array}$ & 162 \\
\hline Oct4 & $\begin{array}{l}\text { F: TCGAGAACCGAGTGAGAGGC } \\
\text { R: CACACTCGGACCACATCCTTC }\end{array}$ & 121 \\
\hline Sox2 & $\begin{array}{l}\text { F: CACACTGCCCCTCTCACACAT } \\
\text { R: CATTTCCCTCGTTTTTCTTTGAA }\end{array}$ & 81 \\
\hline Nanog & $\begin{array}{l}\text { F: CCAACATCCTGAACCTCAGCTAC } \\
\text { R: GCCTTCTGCGTCACACCATT }\end{array}$ & 121 \\
\hline Vimentin & $\begin{array}{l}\text { F: ATGCGTGAGATGGAAGAGAATTTTGC } \\
\text { R: TTATTCAAGGTCATCGTGATGCTGAGA }\end{array}$ & 372 \\
\hline E-Cadherin & $\begin{array}{l}\text { F: TCGACACCCGATTCAAAGTGG } \\
\text { R: TTCCAGAAACGGAGGCCTGAT }\end{array}$ & 192 \\
\hline Snail & $\begin{array}{l}\text { F: TATGCTGCCTTCCCAGGCTTG } \\
\text { R: ATGTGCATCTTGAGGGCACCC }\end{array}$ & 143 \\
\hline $\mathrm{N}$-Cadherin & $\begin{array}{l}\text { F: CCGGAGAACAGTCTCCAACTC } \\
\text { R: CCCACAAAGAGCAGCAGTC }\end{array}$ & 156 \\
\hline GAPDH & $\begin{array}{l}\text { F: TCTGCTCCTCCTGTTCGACA } \\
\text { R: AAAAGCAGCCCTGGTGACC }\end{array}$ & 141 \\
\hline
\end{tabular}

Immunofluorescence analysis of the expression of E-cadherin and vimentin in SP and NSP cells. Fresh-sorted cells were grown on sterile glass cover slides overnight at $37^{\circ} \mathrm{C}$, washed twice with PBS, and fixed in $4 \%$ paraformaldehyde for $30 \mathrm{~min}$ at $4^{\circ} \mathrm{C}$. The cells were washed twice more with PBS, and incubated with $5 \%$ bovine serum albumin for $2 \mathrm{~h}$ at room temperature to block nonspecific binding of $\mathrm{IgG}$. After twice further PBS washes, the cells were incubated overnight at $4^{\circ} \mathrm{C}$ with primary antibodies against E-cadherin and vimentin (BD Biosciences) that had been diluted in PBS according to the manufacturer's instructions. Subsequently, the slides were washed twice with PBS containing 0.02\% Tween-20 (PBS-T) and added fluorochrome-conjugated secondary antibodies (BD Biosciences, USA) at room temperature for $2 \mathrm{~h}$ in a dark chamber. The cells were washed thrice with PBS-T and cover slipped with aqueous mounting medium (containing $0.5 \mathrm{mg} / \mathrm{ml} \mathrm{4,6-diamidino-2-phenylindole} \mathrm{to} \mathrm{stain} \mathrm{the} \mathrm{nuclei).}$ Random photos (Leica TCS SP5II, Germany) of eight visual fields were acquired using 100-fold magnification. The total and positive cell numbers were counted in order to calculate the expression percentage.

Matrigel-Transwell invasion assay in vitro. Matrigel invasion chambers (BD Biosciences) were used to compare the invading capacity between SP and NSP cells. The first step was to prepare artificial basilar membrane. Matrigel was thawed (Sigma, USA) at $4^{\circ} \mathrm{C}$ overnight. Matrigel was diluted (1:3) in serum-free cold RPMI-1640. Diluted Matrigel (100 $\mu \mathrm{l} /$ well) was gently added to ensure homogenicity. The Transwell was incubated at $37^{\circ} \mathrm{C}$ for $3 \mathrm{~h}$ for gelling. SP or NSP fresh sorted cells were collected, washed and resuspended in RPMI-1640 culture medium (containing 2\% FBS) at a density of $5 \times 10^{5}$ cells $/ \mathrm{ml}$. The gelled Matrigel was gently washed with warmed serum free-culture media. RPMI-1640 (600 $\mu \mathrm{l})$ supplemented with $10 \%$ FBS was added to the lower compartments according to the manufacturer's instructions. Cell suspensions $(100 \mu \mathrm{l})$ were respectly put into the upper compartments. Then the Transwell was kept for $48 \mathrm{~h}$ under standard culture conditions. After incubation, the Transwell was removed from 24-well plates and were scrapped off the non-invading cells on the top of the Transwell with a cotton swab. The Transwells were fixed with $95 \%$ alcohol for $15 \mathrm{~min}$ and then stained with crystal violet for $15 \mathrm{~min}$. Images (Nikon, TE2000-S, Japan) of 5 visual fields per membrane of triplicate membranes were captured using 200-fold magnification and the total cells were counted. The test was repeated three times.

Statistical analysis. SPSS 17.0 was used for statistical data analyses. All data were presented as mean \pm SEM from at least three independent experiments. The paired t-test was used for analyzing the difference in colony formation efficiency, Transwell invasion assay and gene expression between the SP and NSP cells. P-values $<0.05$ were considered to indicate significant differences.

\section{Results}

FACS analysis and cell sorting. When examined using FACS, the small population of CNE-2 cells which had the strongest dye efflux ability in the left lower quadrant of the FACS, were gated as SP cells (P3 gate). The main population of cells which were Hoechst 33342 positive were gated as NSP cells (P4 gate). SP cells occupied $16.2 \%$ of the total cells (Fig. 1A). In the control group with verapamil, the percentage of SP 


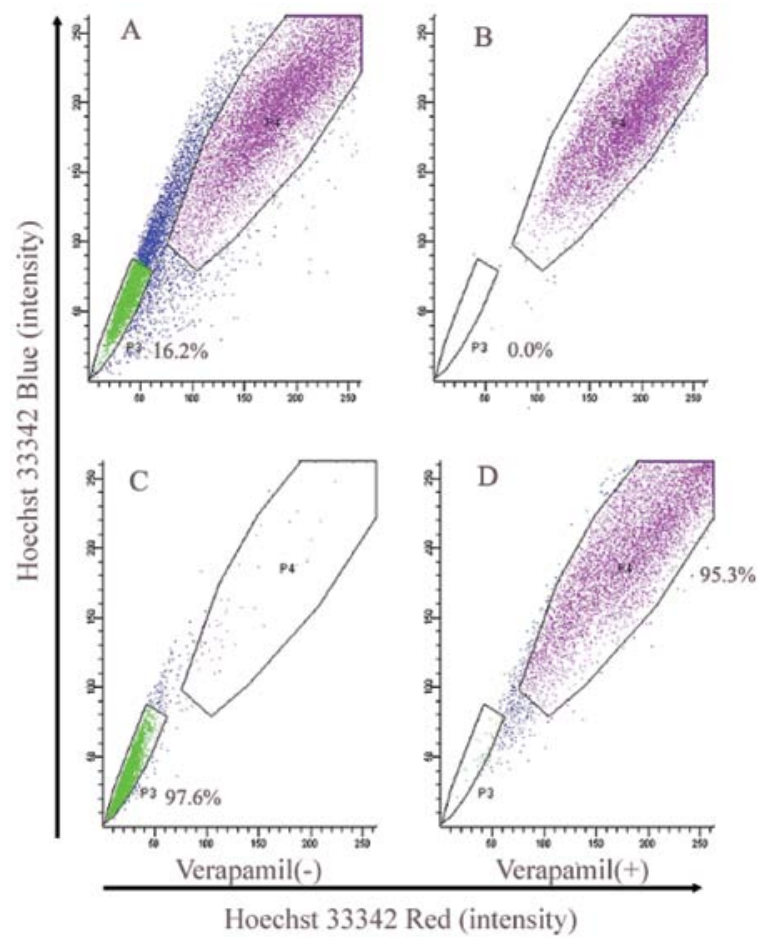

Figure. 1. Cell sorting results and sorting purity. (A) Sorting of CNE-2 cells using Hoechst 33342 . The P3 gate represents the SP cells (16.2\% of total cells) and the P4 gate represents the NSP cells. (B) The SP proportion was decreased to $0.0 \%$ when the cells were preincubated with verapamil to block the ATP transporter. (C and D). The sorting purity of the fresh sorted SP and NSP cells was $97.6 \%$ and $95.3 \%$ respectively.

A

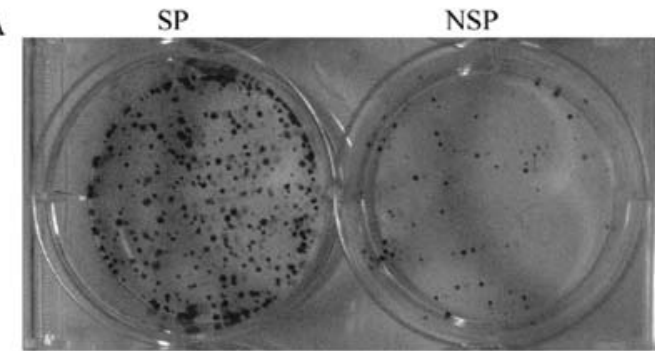

B

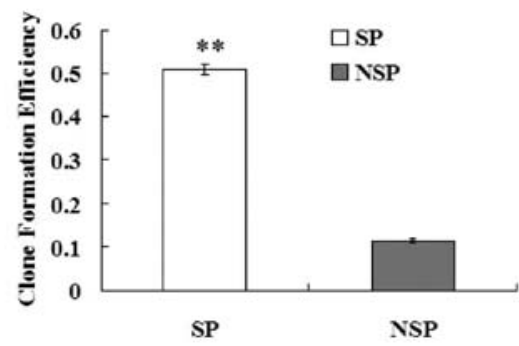

Figure. 2. Results of colony formation assay. (A) SP cells could form more and generally bigger colonies (left plate) compared to NSP cells (right plate). The Clone formation efficiency of the SP cells was significantly higher than that of the NSP cells (B) ( ${ }^{* *} \mathrm{P}<0$.001).

cells dropped to $0.0 \%$ of the total cell population (Fig. 1B), which is consistent with reports that Hoechst 33342 exclusion is verapamil sensitive. The purity of collected SP (P3) and NSP (P4) after sorting were $97.6 \%$ and $95.3 \%$ respectively (Fig. 1C and D), indicating that these cells qualified for further study.
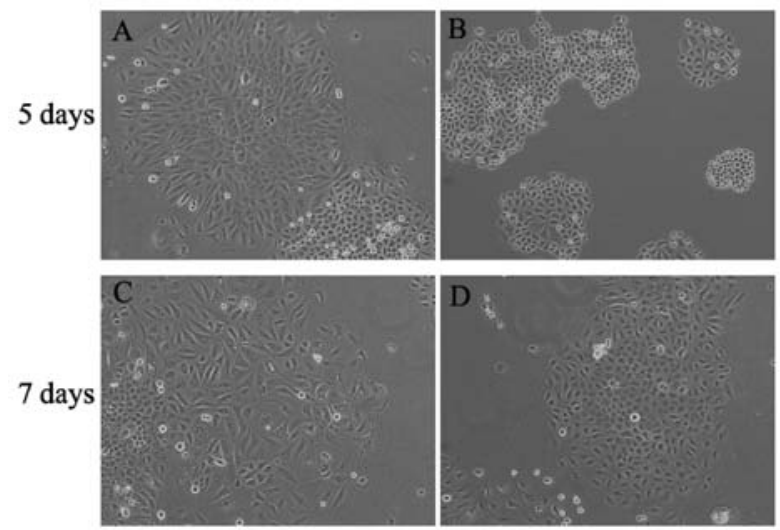

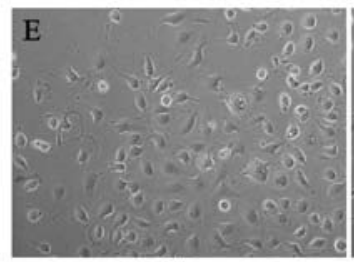

SP

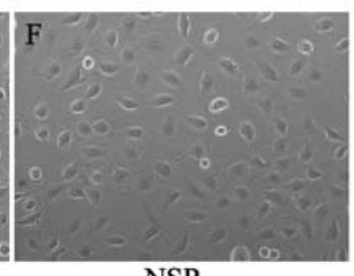

NSP
Figure. 3. Morphological changes of SP and NSP cells in RPMI-1640 and UltraMEM culture; (A and B) SP and NSP cells cultured 5 days in RPMI-1640; Larger clone formation in SP cells and polygonal squamous epithelial cells began to change to fusiform cells (A); (C and D) SP and NSP cells cultured for 7 days in RPMI-1640; SP cells grew many long filaments, causing them to resemble fibroblasts (C); (E and F) SP and NSP cells in UltraMEM culture; SP cells lost their normal appearance and began to extend fibroblast-like filaments (E); NSP cells also displayed morphological changes, although these were not as noticeable as those of SP cells (F).

Colony formation assay. Colony formation assays were repeated thrice in triplicate. After culture for about 12 days at which time most clones reached more than 50 cells, we counted the clone numbers and found that the mean CFE of SP cells $(50.8 \pm 1.06 \%)$ was much higher compared to that of the non-SP cells $(11.4 \pm 0.4 \%)$. A t-test was performed in SPSS 17.0 and there were significant differences in CFE between them, $(\mathrm{P}<0.001)$ (Fig. 2).

Cell morphological changes assay. We seeded the freshsorted SP and NSP cells in RPMI-1640 (with 10\% FBS) and UltraMEM (with 5\% FBS) for contrast and observed the cell morphologic changes dynamically. We found that SP cells grew faster than NSP cells in RPMI-1640 and the formed clones were greater and larger compared to those of NSP cells (Fig. 3A and B). As the cultured time increased, the SP cells began to change shape, from polygonal squamous epithelial cells to fusiform cells and grew many long filaments, causing them to resemble fibroblasts (Fig. 3C), whereas the NSP cells did not change to a greater extent. When maintained in UltraMEM, SP cells grew faster than NSP cells (Fig. 3E and F). Moreover, cell morphology changed markedly in UltraMEM (Fig. 3E and F). SP cells lost their normal appearance in UltraMEM culture and began to extend fibroblast-like filaments (Fig. 3E). NSP cells also displayed morphological changes, although these were not as noticeable compared to the SP cells (Fig. 3F).

Quantitative real-time RT-PCR analysis of stemness and EMT related genes. To determine whether SP cells of NPC have the characteristics of stem cells and EMT, we obtained 


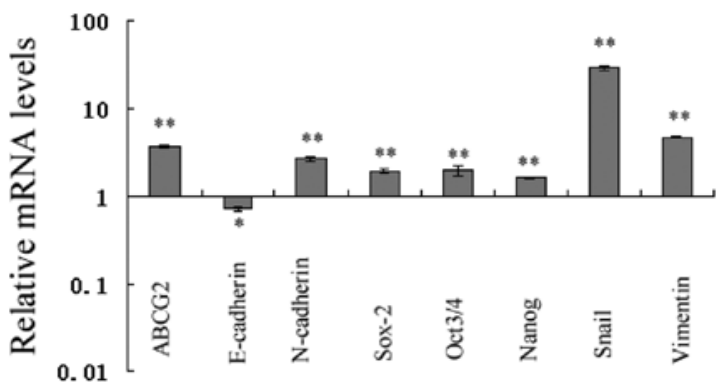

Figure 4. Quantitative PCR analysis of the expression of stemness-related and EMT-related genes. GAPDH mRNA was used to normalize the variability in template loading. The relative expression levels in SP cells to NSP cells were given. The data were reported as mean \pm SEM. Significant differences were ${ }^{*} \mathrm{P}<0.05$ and ${ }^{* *} \mathrm{P}<0.01$. The mRNA levels of stemness-related genes ABCG2, Nanog, Oct $3 / 4, \mathrm{~N}$-cadherin and Sox 2 were increased remarkably in SP cells. The key EMT related genes Snail, $\mathrm{N}$-cadherin and vimentin were also increased in SP cells, whereas E-cadherin was decreased in SP cells.

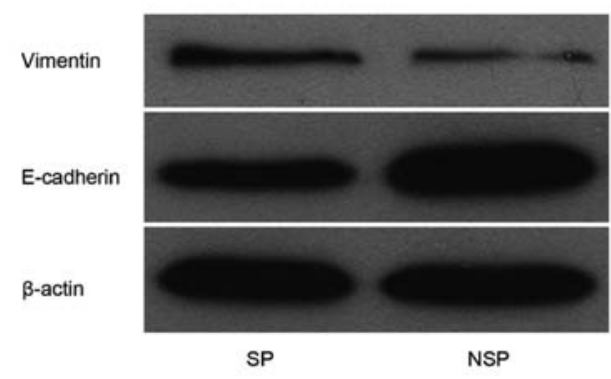

Figure 5. Western blot analysis of E-cadherin and vimentin in the SP cells and NSP cells. A reduced expression of E-cadherin and an increased expression of vimentin in SP cells compared to NSP cells.

RNA from the fresh-sorted SP and NSP cells and analyzed ABCG2, Oct4, Sox2, Nanog, vimentin, E-cadherin, Snail and $\mathrm{N}$-cadherin expression using real-time RT-PCR. Epithelial cell special genen E-cadherin was found to be downregulated in SP cells, whereas the expression levels of the EMT-related genes vimentin, Snail and N-cadherin were higher in SP cells than in NSP cells of NPC. The stemness-related genes, Oct3/4, Sox2, Nanog, ABCG2 were also found to be more highly expressed in SP cells compared to NSP cells (Fig. 4). All differences were statistically significant.

Western blot analysis. Since the enhanced migratory/invasive ability of epithelial cells is often caused by EMT, we analyzed a panel of representative epithelial and mesenchymal markers by immunoblotting to determine whether this process occurs in SP cells of NPC. The results displayed that the SP cells of NPC caused an EMT-like marker shift, which was a dramatic downregulation of the epithelial marker E-cadherin and upregulation of the EMT-associated transcription factor vimentin (Fig. 5).

Immunofluorescence. Immunofluorescence staining further revealed that the expression of E-cadherin dramatically decreased in SP cells contrasting to NSP cells (Fig. 6), whereas the levels of vimentin were strongly increased in SP cells (Fig. 7). These results thus demonstrate that SP cells of NPC have the characteristics of EMT.
Matrigel-Transwell invasion assay in vitro. CSC has the capability to initiate and drive the growth of the primary tumor and of the invasion and metastasis. In analogy to this, we analyzed whether SP cells of NPC could display a similar invasive potential. By using a Matrigel invasion chamber, we compared the invading capacity of SP and NSP cells. The SP cells of the CNE-2 cell line displayed a significantly increased invading capacity $(105.40 \pm 16.118)$ compared to the NSP cells (33.40土10.21) (Fig. 8) ( $\mathrm{P}=0.003)$.

\section{Discussion}

It has been extensively reported that SP cells represent normal stem cells or cancer stem cells in recent years $(13,19,21,22)$. In the present studies we established that SP cells in the nasopharyngeal carcinoma cell line CNE-2 contained SP cells. The ratio of SP cells was on average $16.6 \%$, higher than the ratio reported Wang et al (19), although both studies utilized the same cell line. Many reasons may be causing this difference. As we reported earlier, the SP cell ratio of a cell line is effected by many factors during Hoechst 33342 dyeing, such as cell concentration, Hoechst 33342 concentration and staining time. The same cell line with different passage generations stored in different laboratories may also contain a different SP cell ratio. Although the SP ratio in our study was $16.2 \%$, the cell line was very sensitive to the ABCG2 inhibitor verapamil, which is used to block the ABCG2 pumping Hoechst 33342 function. Corresponding to these results, qRT-PCR further tested that ABCG2 levels in SP cells was much higher compared to NSP cells. ABCG2 is a stem cell marker, and it always has high expression in stem cells, whose function is to efflux many drugs and toxic substances out of the stem cells in order to prolong their lifetime and generate genetic stability. Sox2, Nanog and Oct 4 are transcription factors (TFs) that are always highly expressed in embryonic stem cells, and they are also found to be highly expressed in many cancer stem cells (23-26). In our study, these TFs were more highly expressed in SP cells, indicating that SP cells highly express stemness-related genes. In addition, our results of the colony formation test in vitro indicated that the SP cells had much higher colony formation ability. All these results verified that the SP cells we isolated were enriched with more cancer stem-like cells.

Another capability of CSCs is that they undergo epithelial mesenchymal transition (EMT), a key step during embryogenesis (27-29) and wound healing (30). During EMT, epithelial cells lose their typical epithelial cell shape and change to fibroblast-like cells. Additionally, they break down cell-cell and cell extracellular matrix contacts, and migrate to other locations in the body (14). Evidence suggests that genetic programs relevant to EMT are also transiently activated in epithelial cancer cells through which epithelial cancer cells are able to invade and metastasize. In the present study, we cultured the fresh-sorted SP cells and NSP cells in 10\% FCS medium in a polyHEMA-coated flasks, and found that after several days of culture, some of the SP cells acquired fibroblast-like, mesenchymal appearances, which indicated that SP cells may undergo EMT. Next we cultured sorted SP and NSP cells in an UltraMEM medium containing 5\% FCS, $10 \mathrm{ng} / \mathrm{ml}$ EGF and $5 \mathrm{ng} / \mathrm{ml}$ FGF. Wang et al (19) reported that SP cells grew resembling fibroblasts in this medium. Similar 

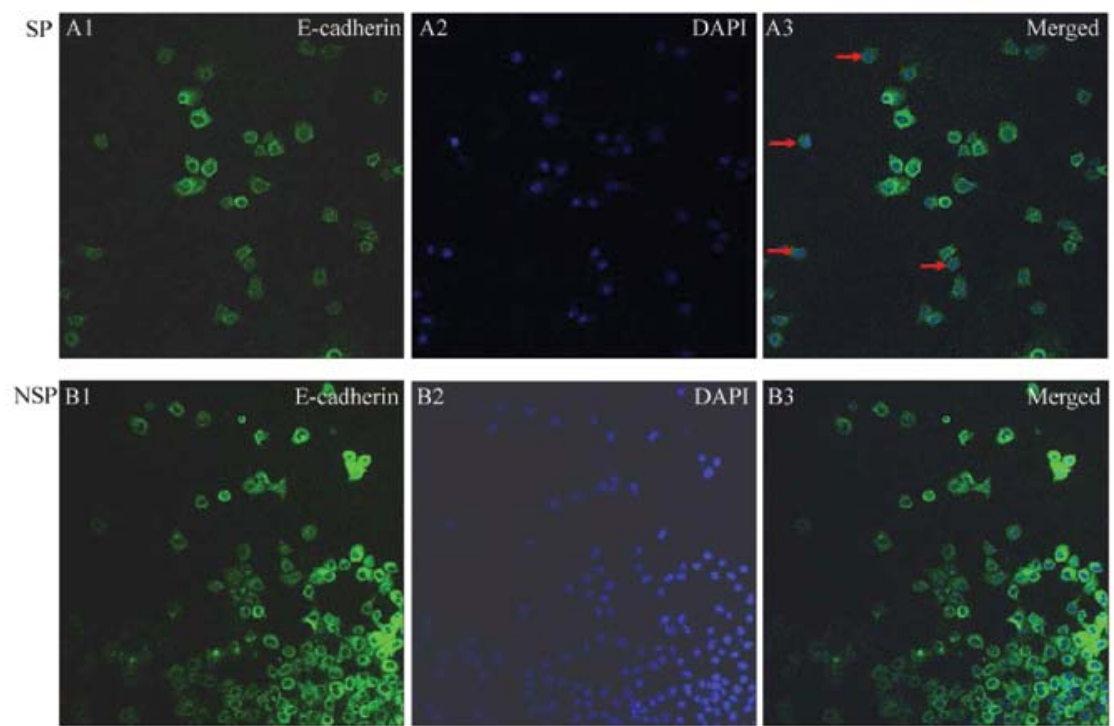

Figure 6. Immunofluorescence images of SP and NSP cells stained using antibody against E-cadherin. Immunofluorescence staining reveals a reduced expression of E-cadherin in SP cells (A1 and A2) in comparison with the NSP cells (B1 and B2)

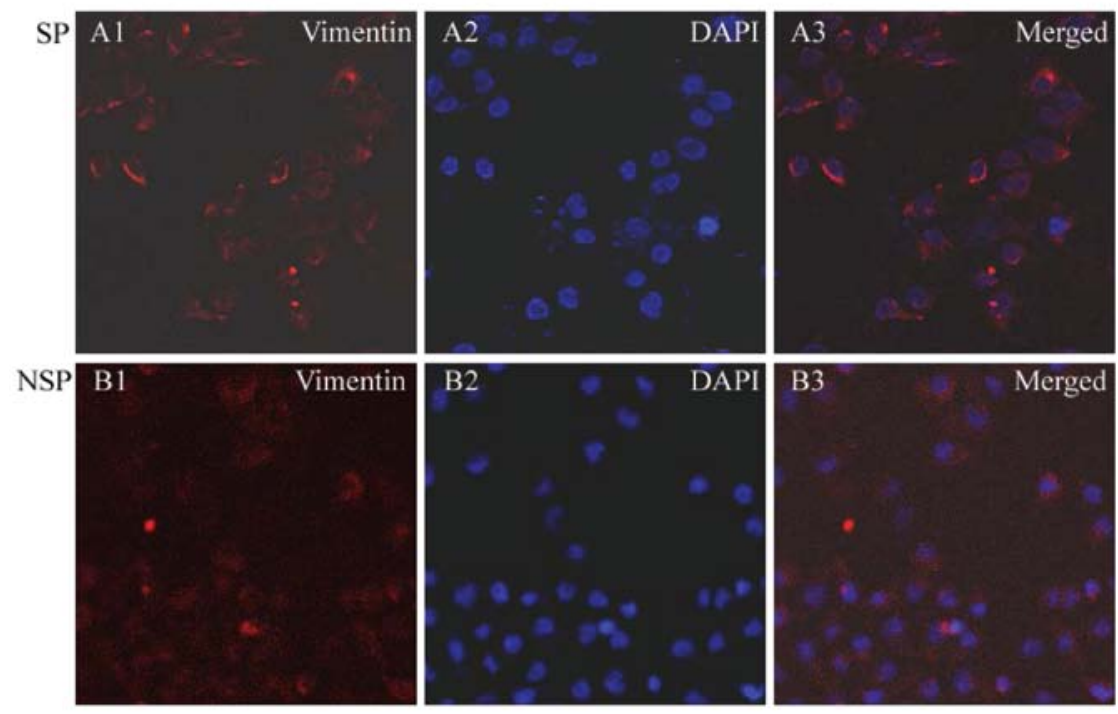

Figure 7. Immunofluorescence images of SP and NSP cells stained using antibody against vimentin. Immunofluorescence staining reveals an increased expression of vimentin in SP cells (A1 and A2) compared to the NSP cells (B1 and B2).
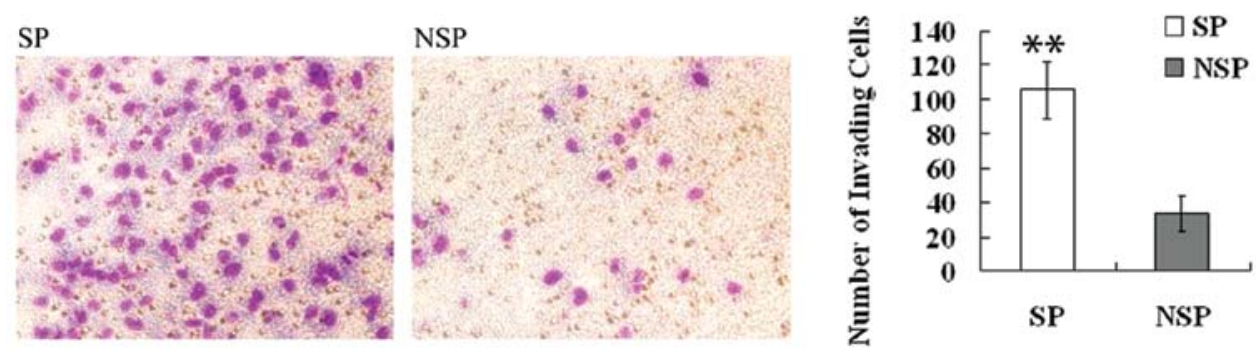

Figure. 8. Invasion assay. Matrigel invasion chambers were used to compare the invading capability between SP and NSP cells. SP cells displays stronger invading activity in vitro than NSP cells $\left({ }^{* *} \mathrm{P}<0.01\right)$

to their results, we also observed that SP cells grew comparable to fibroblast, further confirming the results we observed in the $10 \%$ FCS medium. It has been reported that cancer stem cells possess many mesenchymal characteristics, and they are more amenable to EMT (16). To further test whether SP cells more readily undergo EMT compared to NSP cells, we 
analyzed EMT-related genes expressed in SP and N SP cells by qRT-PCR. Consequently, SP cells expressed higher levels of mesenchymal-related genes $\mathrm{N}$-cadherin, vimentin and Snail, while they expressed low levels of the epithelial-related gene, E-cadherin. These results indicate that genetic programs relevant to EMT are more activated in SP cells. To verify the qRT-PCR results, we performed western blot analysis to confirm the epithelial-typical expressed gene, E-cadherin and the EMT-related gene, vimentin. Consistent with the PCR results, western blot results also demonstrated that E-cadherin was expressed more in NSP cells, and lesser in SP cells; for vimentin, the results were vice versa. When analyzed by immunofluorescence, the expression results of E-cadherin and vimentin were in accordance to western blot analyses. In collaboration, our results support the conclusion that SP cells in the CNE-2 cell line not only enrich cancer stem-like cells, but also possess mesenchymal cell characteristics which make them undergo EMT more readily.

There are several reports indicating how cancer stem cells have the strongest invasion and metastasis ability and how the main cell population causes cancer invasion and metastasis. Cancer metastasis consists of a sequential series of events: the epithelial-mesenchymal transition (EMT) and the mesenchymal-epithelial transition (MET). They are recognized as critical events for metastasis of carcinomas (15). In our present study, we discovered that SP cells had a stronger invading activity compared to NSP cells by using their Matrigel-Transwell invasion assay in vitro, indicating that SP cells may be the main cell population causing nasopharyngeal carcinoma invasion and metastasis. In conclusion, our data indicate that SP cells in CNE-2 cell line possess the properties of cancer stem cells, and they have more mesenchymal cell characteristics related to EMT and cancer cell metastasis. Invasion and metastasis are the main causes leading to death, while EMT is the first step of cancer metastasis. Therefore the study on EMT may be equally important to that of nasopharyngeal carcinoma stem cells.

\section{Acknowledgements}

We thank Dr Yong-Ping Song, Cancer Institute at Henan Cancer Hospital and Ms. Rui-Hua Fan, Laboratory Center of Henan Cancer Hospital for their support.

\section{References}

1. Spano JP, Busson P, Atlan D, Bourhis J, et al: Nasopharyngeal carcinomas: an update. Eur J Cancer 39: 2121-2135, 2003.

2. Ong YK, Heng DM, Chung B, et al: Design of a prognostic index score for metastatic nasopharyngeal carcinoma. Eur J Cancer 39: $1535-1541,2003$.

3. Visvader JE and Lindeman GJ: Cancer stem cells in solid tumours: accumulating evidence and unresolved questions. Nat Rev Cancer 8: 755-768, 2008.

4. Singh SK, Clarke ID, Terasaki M, et al: Identification of a cancer stem cell in human brain tumors. Cancer Res 63: 58215828, 2003.

5. O'Brien CA, Pollett A, Gallinger S, et al: A human colon cancer cell capable of initiating tumour growth in immunodeficient mice. Nature: 445: 106-110, 2007.

6. Collins AT, Berry PA, Hyde C, et al: Prospective identification of tumorigenic prostate cancer stem cells. Cancer Res 65 10946-10951, 2005.
7. Hermann PC, Huber SL, Herrler T, Aicher A, Ellwart JW, et al: Distinct populations of cancer stem cells determine tumor growth and metastatic activity in human pancreatic cancer. Cell Stem Cell 1: 313-323, 2007.

8. Prince ME, Sivanandan R, Kaczorowski A, et al: Identification of a subpopulation of cells with cancer stem cell properties in head and neck squamous cell carcinoma. Proc Natl Acad Sci USA 104: 973-978, 2007.

9. Al-Hajj M, Wicha MS, Benito-Hernandez A, et al: Prospective identification of tumorigenic breast cancer cells. Proc Natl Acad Sci USA 100: 3983-3988, 2003.

10. Zhang S, Balch C, Chan MW, et al: Identification and characterization of ovarian cancer-initiating cells from primary human tumors. Cancer Res 68: 4311-4320, 2008.

11. Shmelkov SV, Butler JM, Hooper AT, et al: CD133 expression is not restricted to stem cells, and both $\mathrm{CD} 133^{+}$and $\mathrm{CD} 133$ metastatic colon cancer cells initiate tumors. J Clin Invest 118: 2111-2120, 2008

12. Mack B and Gires O: CD44s and CD44v6 expression in head and neck epithelia. PLoS One 3: e3360, 2008.

13. Goodell MA, Brose K, Paradis G, Conner AS and Mulligan RC: Isolation and functional properties of murine hematopoietic stem cells that are replicating in vivo. J Exp Med 183: 1797-1806, 1996.

14. Radisky DC and LaBarge MA: Epithelial-mesenchymal transition and the stem cell phenotype. Cell Stem Cell 2: 511-512, 2008.

15. Thiery JP and Sleeman JP: Complex networks orchestrate epithelial-mesenchymal transitions. Nat Rev Mol Cell Biol 7: 131-142, 2006.

16. Mani SA, Guo W, Liao MJ, et al: The epithelial-mesenchymal transition generates cells with properties of stem cells. Cell 133: 704-715, 2008.

17. Chao C, Yan W, Michael H, et al: Evidence for epithelialmesenchymal transition in cancer stem cells of head and neck squamous cell carcinoma. PLoS One 6: e16466, 2011.

18. Yang L, Ping YF, Yu X, et al: Gastric cancer stem-like cells possess higher capability of invasion and metastasis in association with a mesenchymal transition phenotype. Cancer Lett 310: 46-52, 2011.

19. Wang J, Guo LP, Chen LZ, Zeng YX and Lu SH: Identification of cancer stem cell-like side population cells in human nasopharyngeal carcinoma cell line. Cancer Res 67: 3716-3724, 2007.

20. Livak KJ, Schmittgen TD: Analysis of relative gene expression data using real-time quantitative PCR and the 2(-Delta Delta C(T)) Method. Methods 25: 402-408, 2001.

21. Scharenberg CW , Harkey MA and Torok-Storb B: The ABCG2 transporter is an efficient Hoechst 33342 efflux pump and is preferentially expressed by immature human hematopoietic progenitors. Blood 99: 507-512, 2002.

22. Alvi AJ, Clayton H, Joshi C, et al: Functional and molecular characterization of mammary side population cell. Breast Cancer Res 5: R1-R8, 2003.

23. Boyer LA, Lee TI, Cole MF, et al: Core transcriptional regulatory circuitry in human embryonic stem cells. Cell 122: 947-956, 2005.

24. Loh YH, Wu Q, Chew JL, et al: The Oct4 and Nanog transcription network regulates pluripotency in mouse embryonic stem cells. Nat Genet 38: 431-440, 2006.

25. Rodda DJ, Chew JL, Lim LH, et al: Transcriptional regulation of Nanog by OCT4 and SOX2. J Biol Chem 280: 24731-24737, 2005.

26. Chew JL, Loh YH, Zhang W, et al: Reciprocal transcriptional regulation of Pou5f 1 and Sox 2 via the Oct $4 /$ Sox 2 complex in embryonic stem cells. Mol Cell Biol 25: 6031-6046, 2005.

27. Hay ED: An overview of epithelio-mesenchymal transformation. Acta Anat (Basel) 154: 8-20, 1995.

28. Perez-Pomares JM and Munoz-Chapuli R: Epithelialmesenchymal transitions: a mesodermal cell strategy for evolutive innovation in Metazoans. Anat Rec 268: 343-351, 2002.

29. Thiery JP: Epithelial-mesenchymal transitions in development and pathologies. Curr Opin Cell Biol 15: 740-746, 2003.

30. Savagner P, Kusewitt DF, Carver EA, et al: Developmental transcription factor slug is required for effective re-epithelialization by adult keratinocytes. J Cell Physiol 202: 858-866, 2005. 